\title{
Simulation of Alternative Differential Multi-pulse Voltammetry. Evaluation of the Electrochemical Reversibility by the Voltammogram Symmetry
}

\author{
Dijana Jadreško
}

\author{
Division for Marine and Environmental Research, Ruđer Bošković Institute, P.O. Box 180, HR-10002 Zagreb, Croatia \\ Author's e-mail address: djadresko@irb.hr
}

RECEIVED: February 08, 2019 * REVISED: May 09, 2019 * ACCEPTED: May 15, 2019

Abstract: A theoretical analysis of reversible and kinetically controlled electrode reactions in conditions of alternative differential multi-pulse voltammetry (ADMPV) is presented. The degree of reversibility, as well as symmetry of the electron transfer reaction, can be estimated by visual inspection of the ADMP voltammogram. The values of electron transfer coefficient and the standard rate constant of a simple electrode reaction $\mathrm{Ox}+n \mathrm{e}^{-} \rightleftarrows$ Red, can be determined from the slope of linear dependence of the peak currents ratio on the logarithm of pulse duration. The criteria for recognition of reversible and kinetically controlled electrode reactions by alternative differential multi-pulse voltammetry are given.

Keywords: alternative differential multi-pulse voltammetry, excitation signal, kinetics, symmetry, theory.

\section{INTRODUCTION}

$\mathbf{M}$ ETHODOLOGICAL development and application of various pulse techniques for the study/interrogation of electrode reactions have been proposed over the years. ${ }^{[1-3]}$ The main characteristic of modern pulse voltammetric techniques is a stepwise change of the electrode potential to which potential pulses are superimposed with current sampling at the end of each step. ${ }^{[4,5]}$ In such a way, reduction of the charging current and consequently lowering of detection limits were achieved. Accordingly, square-wave voltammetry (SWV) and differential pulse voltammetry (DPV) are the most commonly used pulse techniques for quantitative and qualitative analysis, mechanistic studies and kinetic measurements of the electrode processes. ${ }^{[5-7]}$

However, during the last 20 years, several further variants of (double) potential pulse techniques, as a valuable extension/supplement to the existing ones have been proposed. ${ }^{[3]}$ Accordingly, Molina et al. ${ }^{[8]}$ developed the double pulse potential technique additive differential pulse voltammetry (ADPV) based on recording two (cathodic and anodic) differential pulse voltammograms and plotting their sum versus the first applied potential. They presented the theoretical study of ADPV and its experimental application for different electrode processes. ${ }^{\left[{ }^{[-14]}\right.}$ It has been shown that in ADPV the charging current is minimized to a large extent with respect to "traditional" DPV. A few years later, Zlatev et al. ${ }^{[15]}$ proposed a new electrochemical technique, with a similar name, differential alternative pulse voltammetry (DAPV) based on the non-linearity of $I / E$ characteristic of the electrochemical system. They showed that DAP voltammetry has a higher resolution power and sensitivity compared to some other pulse voltammetric techniques and that is suitable for simultaneous determination of species as well as for multicomponent analysis, without their preliminary separation. ${ }^{[15-19]}$ This technique is characterized by stepping electrode potential to which two rectangular pulses with opposite polarity are superimposed. However, to the best of my knowledge, articles about alternative differential multi-pulse voltammetry (ADMPV) in studying the reversibility and kinetics of a simple charge transfer reaction have not been published yet. 
Table 1. List of symbols

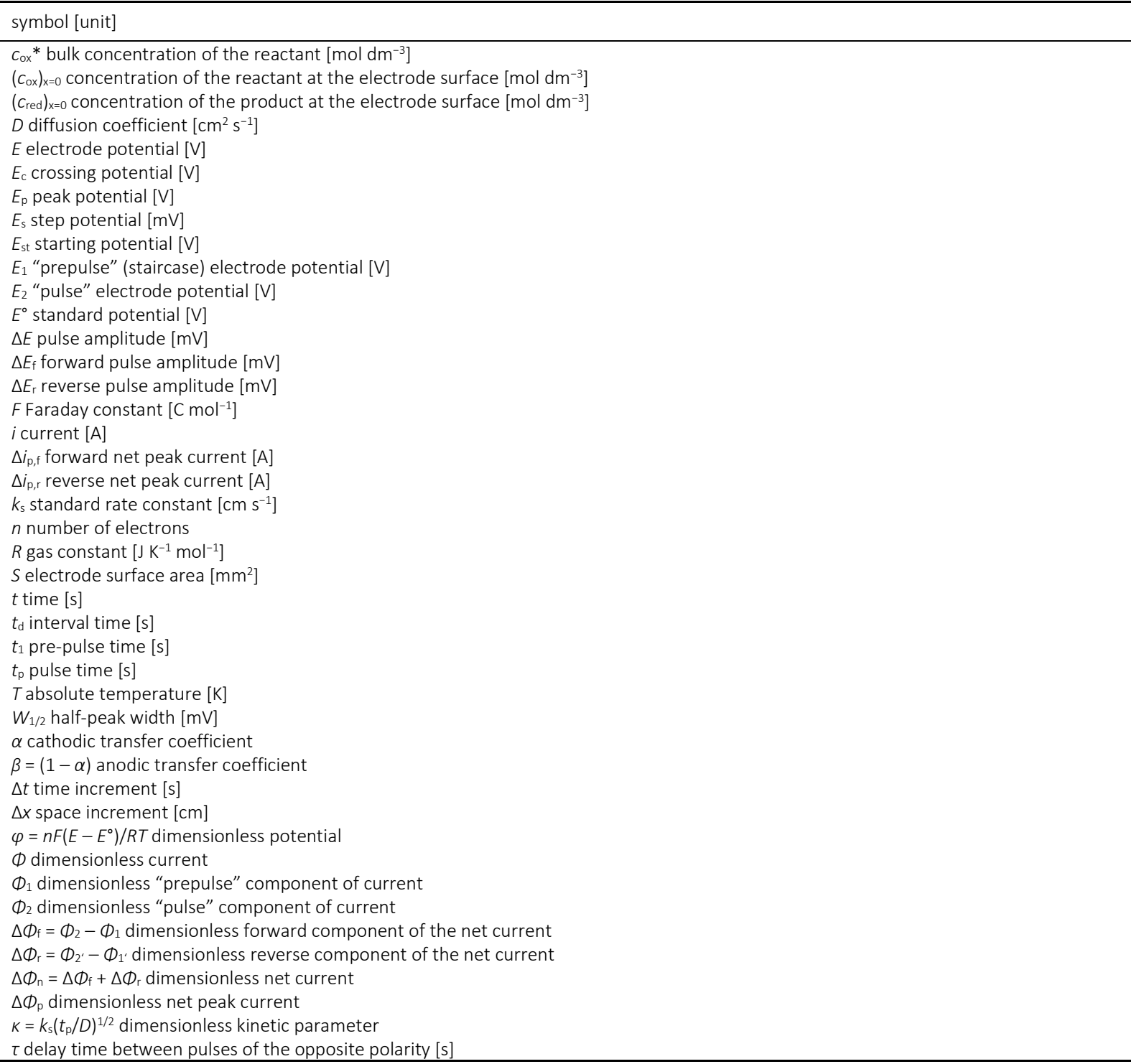

In this article, a multi-pulse technique with specified advantages, i.e. a version of aforementioned techniques that unifies the abilities of SWV to provide mechanistic and kinetic information with the analytical performances of DPV, is proposed. The potential-time program is similar to that employed in $A D P{ }^{[8-14]}$ and $D A P V,{ }^{[15-19]}$ respectively, with the key difference that the initial equilibrium conditions are only restored at the end of the experiment. Thus, the alternative differential multi-pulse voltammetry is expected to enable faster electrochemical measure-ments relative to ADPV and DAPV. The proposed metho-dology is tested by theoretical analysis of the responses of a simple reversible and kinetically controlled electrode reactions, at a planar macroscopic electrode of a solution resident redox couple, where both forms are chemically stable species. However, although ex- perimental confir-mation of this technique is not possible at this time using commercially available instruments/potentiostats and software packages, it is not excluded that everfaster development of technology will soon enable the application of this technique in experimental work. Thus, the existing theories of the above-mentioned techniques (ADPV and DAPV) ${ }^{[8-19]}$ are complemented by giving the working ADMPV curves calculated for standard experimental conditions.

In addition, the theoretical investigation of the influences of timing/kinetic parameters on the ADMPV responses (i.e. characteristic potentials, height and shape of the signal) under conditions of linear diffusion, are also studied. From such analyses, simple diagnostic criteria for elucidation of the reversibility/kinetics of electrode reactions taking place at the planar electrode are given. 

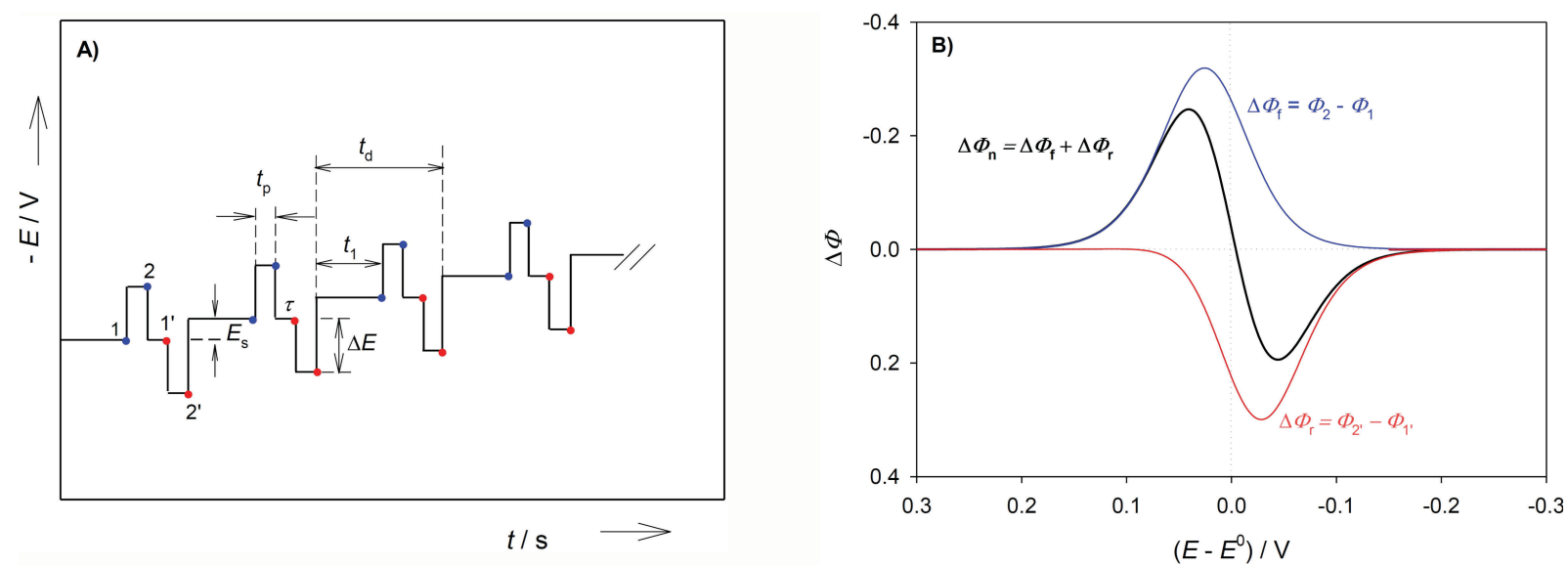

Figure 1. A) Excitation signal in alternative differential multi-pulse voltammetry (ADMPV). Sampling points are schematically indicated for pulse (2) and pre-pulse (1) currents. $E s=$ step potential, $t d=$ interval time, $t p=$ pulse time, $\Delta E=$ pulse amplitude, $\tau=$ delay time between pulses of the opposite polarity. B) Theoretical alternative differential multi-pulse voltammogram (along with the differential "forward" $(\Delta \Phi f)$ and "reverse" $(\Delta \Phi \mathrm{r})$ curves) of reversible electrode reaction (1). Est $=0.3 \vee v s . E^{\circ}, E s=-2$ $\mathrm{mV}, n=1, t \mathrm{~d}=0.3 \mathrm{~s}, t \mathrm{p}=0.05 \mathrm{~s}, \tau=0.05 \mathrm{~s}, \Delta E=50 \mathrm{mV}$.

\section{THE MODEL}

A simple, reversible or kinetically controlled electron transfer reaction on the stationary, planar electrode is considered:

$$
\mathrm{Ox}+n \mathrm{e}^{-} \rightleftarrows \mathrm{Red}
$$

It is assumed that both the reactant $\mathrm{Ox}$ and the product Red are soluble in the aqueous electrolyte phase and are not adsorbed on the electrode surface. Initially, only the reactant is present in the solution. The mass transport is solved by digital simulation, using a common dimensionless diffusion coefficient $D \Delta t / \Delta x^{2}=0.4$, where $\Delta t$ and $\Delta x$ are time and space increments, respectively. ${ }^{[20]}$ The meanings of all symbols are listed in Table 1 . The time increment is defined as: $\Delta t=t_{\mathrm{p}} / 25$.

The kinetics of electrode reaction (1) generally depends on the cathodic transfer coefficient $\alpha$ and the dimensionless kinetic parameter $k=k_{s}\left(t_{p} / D\right)^{1 / 2}$. The conditions at the electrode surface are defined by Nernst (in the case of reversible electrode reaction):

$\left(c_{\text {ox }}\right)_{x=0}=\left(c_{\text {red }}\right)_{x=0} \cdot \exp (\varphi)\left(\right.$ where $\left.\varphi=n F\left(E-E^{\circ}\right) / R T\right)$ or ButlerVolmer equation (in the case of a kinetically controlled electron transfer reaction):

$i / n F S=-k_{\mathrm{s}} \cdot \exp (-\alpha \varphi)\left[\left(c_{\mathrm{ox}}\right)_{\mathrm{x}=0}-\left(c_{\mathrm{red}}\right)_{\mathrm{x}=0} \cdot \exp (\varphi)\right]$.

A program written in Quick Basic is available on request.

In alternative differential multi-pulse voltammetry (ADMPV) excitation signal includes continuous stepwise change of the electrode potential, to which couples of rectangular oppositely oriented pulses, separated by a constant delay time $(\tau)$ and having the same values of the pulse amplitude, are superimposed. Currents are measured before $\left(\Phi_{1}, \Phi_{1^{\prime}}\right)$ and at the end $\left(\Phi_{2}, \Phi_{2^{\prime}}\right)$ of each pulse, and the sum of differences $\Delta \Phi_{\mathrm{n}}=\Delta \Phi_{\mathrm{f}}+\Delta \Phi_{\mathrm{r}}=\left(\Phi_{2}-\Phi_{1}\right)+\left(\Phi_{2^{\prime}-}\right.$ $\left.\Phi_{1^{\prime}}\right)$ is plotted against the staircase potential (Figure 1). In a theory, the dimensionless current is calculated: $\Phi=i\left(\pi t_{\mathrm{p}}\right)^{1 / 2}\left(n F S C_{\mathrm{ox}} \times D^{1 / 2}\right)^{-1}$.

\section{RESULTS AND DISCUSSION}

In this paper, the theoretical possibilities of alternative differential multi-pulse voltammetry (ADMPV) for study and recognition of various kinetic and diffusion controlled electrode processes are presented. The proposed technique is based on the potential-time waveform (excitation signal) defined in Figure 1A., which is the main difference compared to techniques described in refs. ${ }^{[8-19]}$ More precisely, in ADMPV excitation signal includes continuous stepwise change of the electrode potential, to which couples of rectangular pulses (opposite polarity) are superimposed. Furthermore, contrary to Zlatev et al.,, ${ }^{[15]}$ in this article, all simulations were performed using shorter delay time between the opposite pulses (see further in text). As a result, the initial equilibrium conditions are not re-established after each potential step/cycle (unlike the results given in Refs. $\left.{ }^{[8-19]}\right)$. However, design of the potential modulation together with the current-sampling procedure of alternative differential multi-pulse voltammetry, enable effective reduction of the charging current, faster (timesaving) analysis and lower detection limits, providing a signal with high resolution power and enhanced sensitivity. Accordingly, the response is well-defined $I-E$ curve, which is very convenient for the quantitative analysis of the system, 

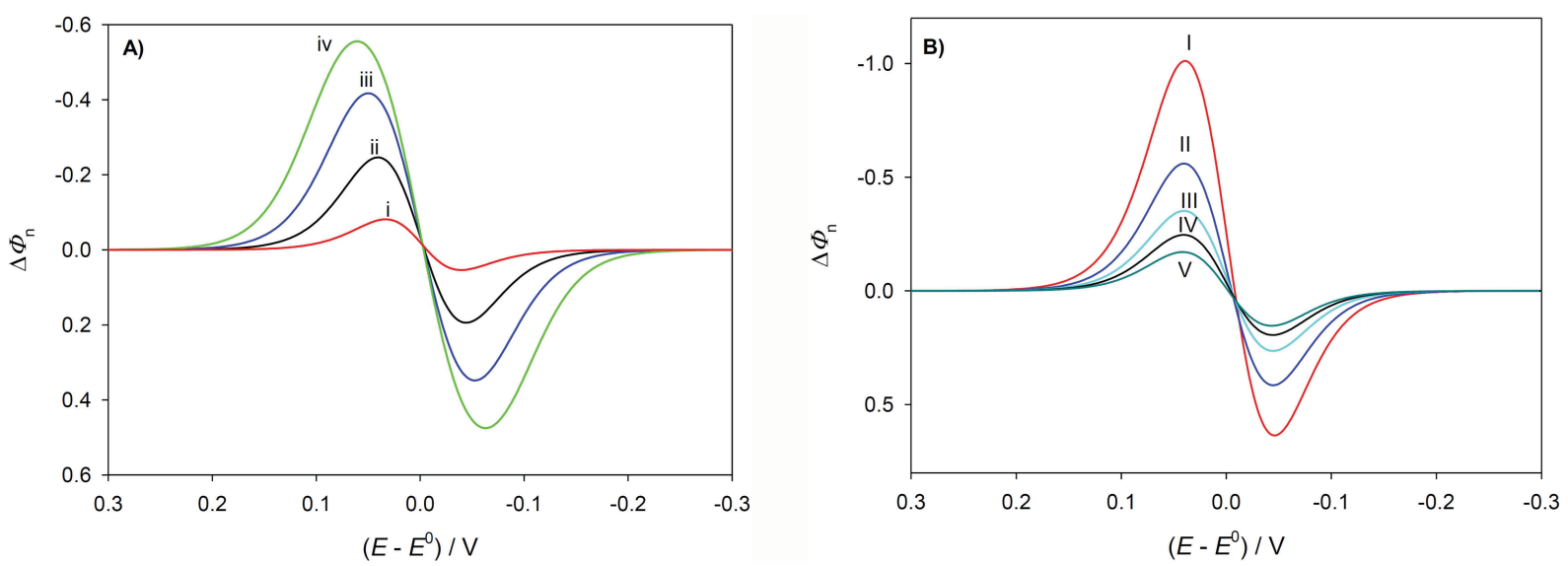

Figure 2. Influence of the $\mathrm{A}$ ) pulse amplitude, $\Delta E$, and $\mathrm{B}$ ) time parameters, tp and $\tau$, on the net ADMPV response, for a reversible electrode reaction (1). Est $=0.3 \mathrm{~V} v \mathrm{~s}$. $E^{\circ}, E s=-2 \mathrm{mV}, n=1, t \mathrm{~d}=0.3 \mathrm{~s}$. A) $t \mathrm{p}=0.05 \mathrm{~s}, \tau=0.05 \mathrm{~s}, \Delta E / \mathrm{mV}=25$ (i), 50 (ii), 75 (iii) and 100 (iv). B) $\Delta E=50 \mathrm{mV}, \mathrm{tp}(=\tau) / \mathrm{ms}=5$ (I), 10 (II), 25 (III), 50 (IV) and 100 (V).

as well. Furthermore, from Figure $1 \mathrm{~A}$ it can be seen that during each potential step/cycle currents are measured four times, twice before $\left(\Phi_{1}\right.$ and $\left.\Phi_{1^{\prime}}\right)$ and twice at the end $\left(\Phi_{2}\right.$ and $\left.\Phi_{2^{\prime}}\right)$ of each pulse, while the sum of their differences $\left(\Delta \Phi_{\mathrm{n}}=\left(\Phi_{2}-\Phi_{1}\right)+\left(\Phi_{2^{\prime}}-\Phi_{1^{\prime}}\right)\right)$ is plotted against the staircase potential (Figure 1B) (as in the classical DPV and SWV). ${ }^{[5-7]}$ Thus, one of the advantages of a given technique is the fact that during a single potential cycle, the electrode reaction is driven in both cathodic and anodic directions, providing an insight into the electrode mechanism. More precisely, the obtained data can be presented in a form of forward/cathodic and reverse/anodic ADMPV curve (Figure 1B) (same as in $\left.S W V{ }^{[6]}\right)$. Furthermore, from Figure $1 B$ (black curve) it can be seen that the maximum current of forward/reduction peak is somewhat higher than the reverse/oxidation one (i.e. $\left.\left|\Delta \Phi_{\mathrm{p}, \mathrm{r}} / \Delta \Phi_{\mathrm{p}, \mathrm{f}}\right|=0.79\right)$ as a result of the concentration gradient of redox components in diffusion layer near the electrode surface. These observations are different from the results given by Laborda et al..12,13] where $\left|\Delta \Phi_{\mathrm{p}, \mathrm{r}}\right|$ $\Delta \Phi_{\mathrm{p}, \mathrm{f}} \mid=1$ and $E_{\mathrm{c}}=E^{\circ}$ (in case of a reversible redox reaction) as a result of different potential-time waveforms. More precisely, in ADMPV at the "middle-pulse" potential, the product Red generated during the forward/cathodic pulse diffuses away during the delay time $(\tau)$ separating the two pulses with opposite polarities. As a result, its concentration on the electrode surface is decreased and the current response due to the anodic pulse(s) is smaller, i.e. $\left|\Delta \Phi_{p, r} / \Delta \Phi_{p, f}\right|<1$. This statement applies to the examined/standard parameters $\left(n=1, t_{\mathrm{d}}=0.3 \mathrm{~s}, t_{\mathrm{p}}=50 \mathrm{~ms}\right.$, $\left.\tau=50 \mathrm{~ms}, E_{\mathrm{s}}=-2 \mathrm{mV}, \Delta E=(-) 50 \mathrm{mV}, E_{\mathrm{st}}=0.3 \mathrm{Vvs} . E^{\circ}\right)$. In general, renewal of the initial conditions is only achieved by waiting long enough at "pre- /middle- pulse" potential, i.e. both peak currents gain the same absolute value (i.e.
$\left|\Delta \Phi_{\mathrm{p}, \mathrm{r}} / \Delta \Phi_{\mathrm{p}, \mathrm{f}}\right|=1$ ) only if $\tau>2 \cdot \mathrm{tp}$ (in case of a reversible electrode reaction).

Figure 2 shows the dimensionless theoretical alternative differential multi-pulse voltammograms of a simple reversible electrode reaction (1) at the stationary planar electrode, for different values of the pulse amplitude $(\Delta E)$ and of the time parameters ( $t_{\mathrm{p}}$ and $\tau$, if $\tau=$ $\left.t_{p}\right)$. The net ADMPV response consists of two apparently "symmetric" peaks at different sides of the potential axis: a forward/cathodic peak followed immediately by anodic one, which reflect the reduction of the reactant $O x$ and reoxidation of the product Red, respectively. In all cases, ADMPV curves have a center of symmetry at the crossing potential (intercept point with the potential axis) which coincides with a value of the formal potential, i.e. $E_{\mathrm{c}} \approx E^{\circ}$. Furthermore, as can be seen in Figure 2, the $E \mathrm{c}$ value of a simple reversible electrode reaction is independent of the pulse amplitude and of the time parameters. However, the amplitude affects both the peak currents and half-peak widths $\left(W_{1 / 2}\right)$ so that both increase nonlinearly with increasing its value $(\Delta E)$ (Table 2$)$. These are in accordance with the results given in references. ${ }^{[12,15]}$ On the other hand, by increasing the values of $\tau$ and tp simultaneously, both forward/cathodic and reverse/anodic peak currents decrease (Figure 2B). Furthermore, unlike data from Laborda et al.[13] the net ADMP voltammogram is not a result of the addition of two differential pulse voltammograms. More specifically, in ADMP voltammetry forward/cathodic pulse is followed by a short period of time $(\tau)$ at first potential, after which reverse/anodic pulse is applied on the same potential step (see Figure $1 \mathrm{~A}$ ). It is important to note that these empirical parameters ( $t p$ and $\tau$ ) could have the same or different values (i.e. there are many possible combinations of these parameters), on 
Table 2. Alternative differential multi-pulse voltammetry of fast and reversible electrode reaction (1). The dimensionless forward and reverse net peak currents and the corresponding half-peak widths as functions of pulse amplitude. $n=1, E s t=0.3$ $V$ vs. $E^{\circ}, E s=-2 \mathrm{mV}, t \mathrm{~d}=0.3 \mathrm{~s}, \mathrm{tp}=0.05 \mathrm{~s}, \tau=0.05 \mathrm{~s}$

\begin{tabular}{|c|c|c|c|c|}
\hline$\Delta E / \mathrm{mV}$ & $\Delta \Phi_{p, f}$ & $\Delta \Phi_{p, r}$ & $W_{1 / 2, \mathrm{f}} / \mathrm{mV}$ & $W_{1 / 2, r} / m V$ \\
\hline 5 & -0.0046 & 0.002 & 65 & 58 \\
\hline 10 & -0.0165 & 0.0074 & 66 & 60 \\
\hline 20 & -0.0554 & 0.0338 & 66 & 61 \\
\hline 30 & -0.1105 & 0.0774 & 68 & 63 \\
\hline 40 & -0.1760 & 0.1327 & 70 & 66 \\
\hline 50 & -0.2464 & 0.1942 & 74 & 69 \\
\hline 60 & -0.3173 & 0.2573 & 77 & 74 \\
\hline 75 & -0.4174 & 0.3481 & 84 & 82 \\
\hline 100 & -0.5558 & 0.4751 & 99 & 96 \\
\hline 150 & -0.7229 & 0.6299 & 134 & 132 \\
\hline 200 & -0.7947 & 0.6966 & 177 & 176 \\
\hline 300 & -0.8342 & 0.7333 & 274 & 271 \\
\hline
\end{tabular}

which appearance (i.e. apparent symmetry) of voltammogram depends. In this article, all simulations were performed using the same values of the pulse time and of delay time between them (i.e. $t_{p}=\tau$ ) as well as the amplitudes ratio $\left|\Delta E_{\mathrm{f}} / \Delta E_{\mathrm{r}}\right|=1$. This is contrary to data from Zlatev et al. ${ }^{[15]}$ where the value of $\tau \gg t_{\mathrm{p}}$ is used. The described results, as well as the preserved apparent symmetry of the ADMPV response, regardless of the values of examined parameters (i.e. $\Delta E, t_{\mathrm{p}}$ and $\tau$ ), are characteristic of a simple reversible electrode reaction with both components of the redox pair dissolved in the electrolyte solution.

In addition, the influence of the charge transfer kinetics on the theoretical alternative differential multipulse voltammograms was studied. For this reason, the influence of dimensionless kinetic parameter $(\kappa=$ $\left.k_{\mathrm{s}}\left(t_{\mathrm{p}} / D\right)^{1 / 2}\right)$ was investigated for the following set of standard parameters: $t_{\mathrm{d}}=0.3 \mathrm{~s}, t_{\mathrm{p}}=\tau=50 \mathrm{~ms}, \Delta E=(-) 50$ $\mathrm{mV}, E_{\mathrm{s}}=-2 \mathrm{mV}, E_{\mathrm{st}}=0.3 \mathrm{~V} v \mathrm{~s}$. $E^{\circ}$. The simulations were performed for $n=1$ and for various values of the transfer coefficient: $0.1<\alpha<0.9$.

As can be seen from Figure 3, absolute values of both peak currents $\left(\Delta \Phi_{\mathrm{p}, \mathrm{f}}\right.$ and $\left.\Delta \Phi_{\mathrm{p}, \mathrm{r}}\right)$ decrease and the appearance/symmetry of the ADMP voltammogram changes as the value of $\kappa$ decreases. More precisely, the forward (and reverse) half-peak width of the net ADMPV signal, changes from $74 \mathrm{mV}$ (and $69 \mathrm{mV}$ ) for a reversible reaction to $157 \mathrm{mV}$ (and $119 \mathrm{mV}$ ) for an irreversible electrode reaction. In other words, in case of an irreversible electrode reaction, the net ADMP voltammogram consists of a broad cathodic peak and a very low anodic one. Moreover, the reverse/anodic peak of the ADMPV response completely disappears for the values of $k<0.05$. Furthermore, the crossing potential $\left(E_{c}\right)$ shifts to more negative values as the value of $\kappa$ decreases. These effects as well as the loss of the apparent symmetry of the voltammogram and the ratio $\left|\Delta i_{p, r} / \Delta i_{p, f}\right| \neq 0.79$, indicate decreased reversibility of the electrode process.

Figure 4 shows the relationships between the net peak currents ratio as well as crossing potentials of ADMPV response and the logarithm of the kinetic parameter. The linear parts of the curves (1-3) in Figure 4A, are typical for the quasireversible kinetic region (as in $S W{ }^{[6]}$ ), whereas the plateaus for $\log K<-1.5$ and $\log K>0$ correspond to the irreversible and reversible kinetic region, respectively. In other words, in ADMPV the electrode reaction (1) appears reversible (i.e. $\left|\Delta i_{\mathrm{p}, \mathrm{r}} / \Delta i_{\mathrm{p}, \mathrm{f}}\right|=0.79$ and $E_{\mathrm{c}} \approx E^{\circ}$ ) if $\log \kappa \geq 0$. Within the range $-1.5<\log k<-0.5$ the reaction (1) is quasireversible. In this case, if $\alpha<0.5$ the maximum current of forward/cathodic ADMPV peak is lower than the anodic one, so their ratio is $\left|\Delta i_{\mathrm{p}, \mathrm{r}} / \Delta i_{\mathrm{p}, \mathrm{f}}\right|>0.79$, and vice versa if $\alpha \geq$ 0.5 (see Figure $4 A$ ). Thus, from the ADMPV peak currents ratio, it is possible to deduce the energy profile of the electrode reaction i.e. symmetry of the electron transfer, as well as apparent reversibility of the electrode process.

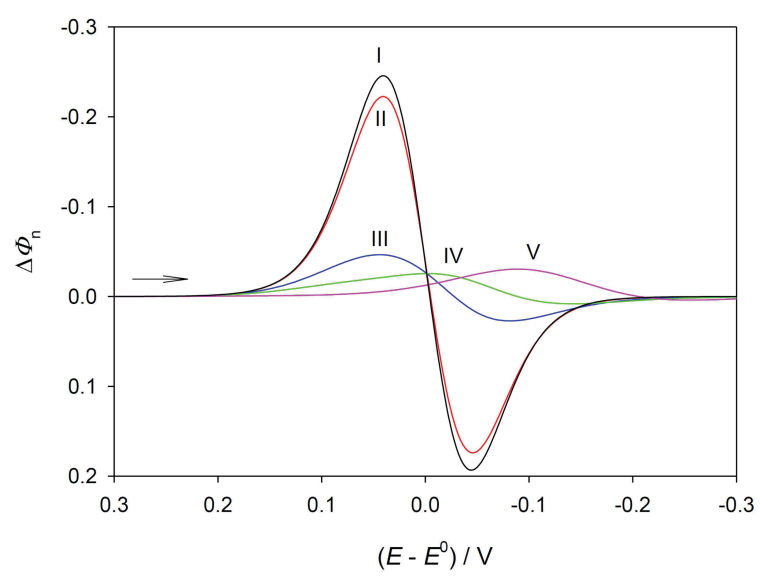

Figure 3. Influence of the dimensionless kinetic parameter, $\kappa$, on theoretical alternative differential multi-pulse voltammograms for $\alpha=0.5 . k=10$ (I), 1 (II), 0.1 (III), 0.05 (IV) and $0.01(\mathrm{~V})$. All other parameters are as in Figure 1. 

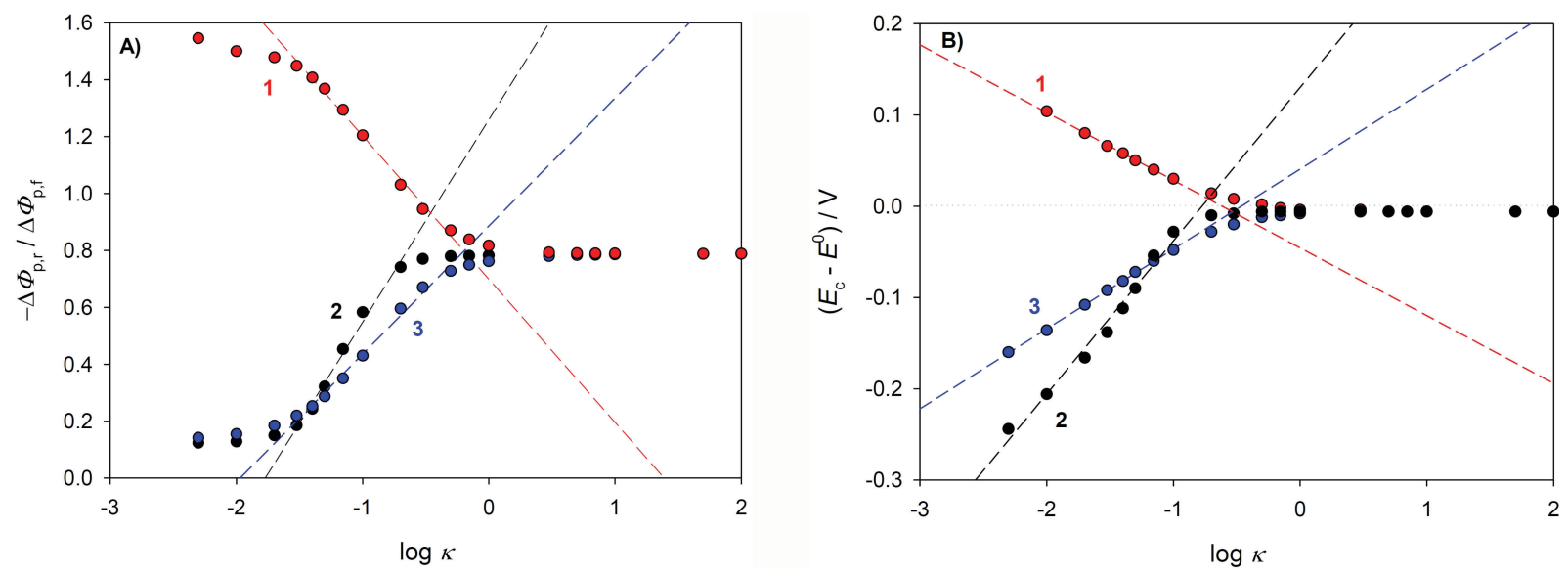

Figure 4. Theoretical dependencies of the A) peak currents ratio and B) crossing potentials of the net ADMP voltammograms on the logarithm of kinetic parameter, for $\alpha=0.25$ (1), 0.5 (2) and 0.75 (3). All other parameters are as in Figure 1.

Besides, Figure 4B implicates that the relationships between the crossing potentials of ADMPV signal and the logarithm of kinetic parameter are linear as well, if $\log K \leq$ -0.5 , i.e.:

$$
\frac{\partial E_{c}}{\partial \log K}=\frac{m}{\alpha n}
$$

where $m$ is a constant i.e. the slope of the straight line, while its "positive" or "negative" sign indicates the value of $\alpha \geq 0.5$ or $\alpha<0.5$, respectively.

Experimentally, the influence of kinetics of electron transfer reaction on the height, shape and characteristic (e.g. crossing and/or peak) potentials of the voltammetric signal can generally be studied by changing the time scale of the experiment. In ADMP voltammetry (as in DPV) this means by changing the pulse time, $t_{\mathrm{p}}$. More precisely, the variation of kinetic parameter, $k$, can be achieved by changing the parameter $t p$, whereas the results are usually presented by plotting the peak currents ratio $\Delta i_{\mathrm{p}, \mathrm{r}} / \Delta i_{\mathrm{p}, \mathrm{f}}$ (or crossing potentials) in dependence of the logarithm of pulse time (Figure 5). Hence, if such (linear) dependence is experimentally obtained for e.g. $-2<\log t_{\mathrm{p}}<-1$, it means that $-1.5 \leq \log \kappa<-0.7$ (for symmetric charge transfer), assuming that other parameters are the same as in Figure $4 \mathrm{~A}$. From this result, it follows a rough estimation of the rate constant of the electron transfer reaction, i.e. $-0.5 \leq$ $\log k_{\mathrm{s}}-1 / 2 \log D<-0.2$, considering that the pulse time and the kinetic parameter are connected by the following equation:

$$
\log K=\log k_{\mathrm{s}}-\frac{1}{2} \log D+\frac{1}{2} \log t_{\mathrm{p}}
$$

(the value of $D$ can be determined from the limiting cathodic/reduction current of the e.g. normal pulse voltammogram, ${ }^{[21,22]}$ by Cottrell equation.) Accordingly, if e.g. $D=1 \times 10^{-6} \mathrm{~cm}^{2} \mathrm{~s}^{-1}$ then $-3.5 \leq \log k_{\mathrm{s}}<-3.2$. However, if experimentally obtained $\left|\Delta i_{\mathrm{p}, \mathrm{r}} / \Delta i_{\mathrm{p}, \mathrm{f}}\right|$ is lower than 0.2 (or greater than 1.4; see Figure $4 \mathrm{~A}$ ), i.e. nearly constant for all pulses shorter than $0.1 \mathrm{~s}$ it means that $\log K<-2$ and $\log k_{\mathrm{s}}$ $<-1.5+1 / 2 \log D$ (i.e. $\log k_{\mathrm{s}}<-4.5$ if $D=1 \times 10^{-6} \mathrm{~cm}^{2} \mathrm{~s}^{-1}$ ) (see Figure 5).

Another possibility for determination of the $k_{\mathrm{s}}$ value arises from the linear part of the crossing potentials vs. log tp plot (as in Figure 4B). In the range of shortest pulse times, in which the electrode reaction is apparently irreversible, the relationship between crossing potentials and the logarithm of pulse duration is linear.

The influence of the transfer coefficient $(\alpha)$ on dimensionless ADMP voltammograms (in case of an irreversible reduction reaction (1) of a dissolved redox

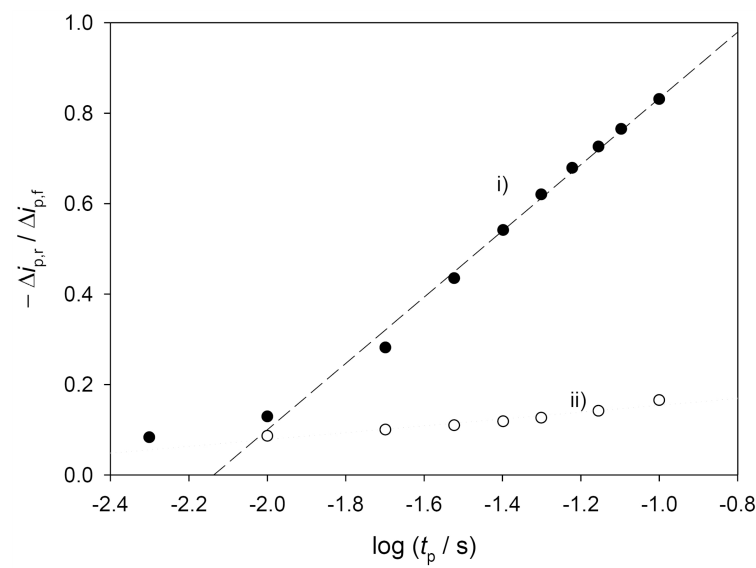

Figure 5. The dependencies of the net ADMPV peak currents ratio on the logarithm of pulse time for two values of the standard rate constant of electrode reaction (1): $\mathrm{ks} / \mathrm{cm} \mathrm{s}^{-1}$ $=5 \times 10^{-4}$ (i) and $3 \times 10^{-5}$ (ii). $\alpha=0.5, \tau=t \mathrm{p}$. All other parameters are as in Figure 1. 


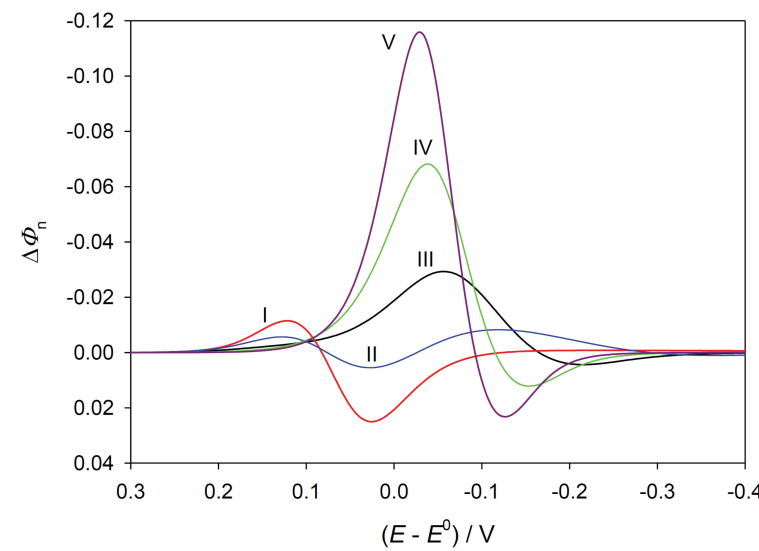

Figure 6. Influence of the electron transfer coefficient on the net ADMP voltammograms of an irreversible electrode reaction (1); $k=0.02, \alpha=0.1$ (I), 0.3 (II), 0.5 (III), 0.7 (IV) and 0.9 (V). All other parameters are as in Figure 1.

couple) is shown in Figure 6. As can be seen, the transfer coefficient affects considerably the appearance (i.e. the shape/symmetry and the characteristic potentials $\left(E_{\mathrm{c}}\right.$ and $\left.E_{\mathrm{p}}\right)$ ) of the ADMPV response. More specifically, the "asymmetry" between the maximum of forward and the minimum of the reverse peak currents increases as the value of $\alpha$ increases. In other words, the ADMP voltammogram of the asymmetric charge transfer $(\alpha=0.1)$, consists of two peaks at different sides of the potential axis (similar as in the case of a reversible electrode process). However, $\left|\Delta \Phi_{\mathrm{p}, \mathrm{r}}\right|>\left|\Delta \Phi_{\mathrm{p}, \mathrm{f}}\right|$ indicates that oxidation is, in term of energy, a more favorable process. Furthermore, in the case of symmetric reduction reaction with slow kinetics (i.e. for $\alpha=0.5$ ), the ADMPV response consists of a broad cathodic peak and a very low anodic one, indicating that the reoxidation is a very slow process. A similar observation is valid for $\alpha>0.5$, and the final conclusion is the same. In addition, contrary to all simulated voltammograms, the anomalous shape of the ADMPV signal for $\alpha=0.3$, which consists of two cathodic and one anodic peak, can be seen. This is in accordance with observation first described by Laborda et al. ${ }^{[12]}$ and further indicates un-symmetric charge transfer as well as decreased reversibility of the electrode process. Thus, the response in ADMPV reflects the symmetry of the electron transfer, i.e. gives qualitative information about the transfer coefficient and apparent rate of the electrode reaction.

Figure 7 shows dependencies of the normalized forward/cathodic and reverse/anodic peak currents $\left(\Delta \Phi_{\mathrm{p}} t_{\mathrm{p}}{ }^{-1 / 2}\right)$ of the ADMP voltammogram on $t_{\mathrm{p}}{ }^{-1 / 2}$ for a simple reversible electrode reaction and for two values of the dimensionless kinetic parameter: $k=0.3$ and 0.02 (corresponding to quasireversible and totally irreversible redox reaction, respectively). Opposite to Laborda et al., ${ }^{[12]}$

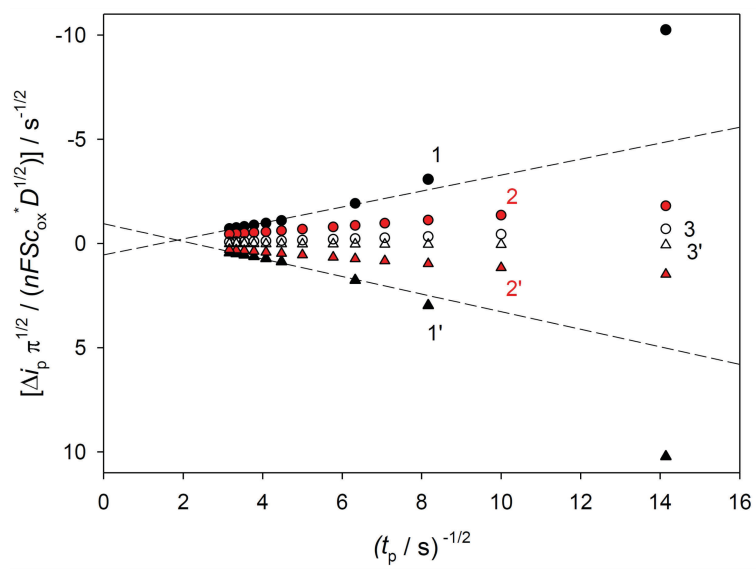

Figure 7. The dependencies of the normalized forward $(\bullet)$ and reverse ( $\boldsymbol{\Delta}$ ) peak currents of the net ADMPV response on $t \mathrm{p}^{-1 / 2}$, for reversible (1) and for two values of the dimensionless kinetic parameter: $k=0.3$ (2) and 0.02 (3). All other parameters are as in Figure 1.

one can see that in the case of kinetically controlled (in contrast to reversible) electrode reactions (1), dependencies of the normalized peak currents of the net ADMP voltammogram on $t_{\mathrm{p}}{ }^{-1 / 2}$ are linear and that the "virtual" slopes of corresponding straight lines decrease as the $\kappa$ value diminishes. The decreased slope indicates a reduced influence of timing parameter $\left(t_{p}\right)$ on ADMP voltammogram, compared with a simple reversible electrode reaction. Moreover, the reduced values of the net peak currents are the result of the concentration gradient of the redox components in diffusion layer, i.e. indicate a decrease of their concentrations near the electrode surface as a consequence of the slow electrode kinetics.

\section{CONCLUSIONS}

The signals that arise from the application of alternative differential multi-pulse voltammetry were simulated. The criteria for recognition of reversible and kinetically controlled electrode reactions are given in Table 3 .

Theoretical analysis indicates that ADMP voltammetry enables easy characterization of the electrode processes. The alternative differential multi-pulse

Table 3. The criteria for recognition of reversible and kinetically controlled electrode reactions

\begin{tabular}{ccc}
\hline \multirow{2}{*}{ Reversible } & \multicolumn{2}{c}{ Non-reversible } \\
\cline { 2 - 3 } & $\alpha<0.5$ & $\alpha \geq 0.5$ \\
\hline$\left|\Delta i_{\mathrm{p}, \mathrm{r}} / \Delta i_{\mathrm{p}, \mathrm{f}}\right|=0.79$ & $\left|\Delta i_{\mathrm{p}, \mathrm{r}} / \Delta i_{\mathrm{p}, \mathrm{f}}\right|>0.79$ & $\left|\Delta i_{\mathrm{p}, \mathrm{r}} / \Delta i_{\mathrm{p}, \mathrm{f}}\right|<0.79$ \\
$E \mathrm{c} \approx E^{\circ}$ & $E_{\mathrm{c}}>E^{\circ}$ & $E_{\mathrm{c}}<E^{\circ}$ \\
\hline
\end{tabular}


voltammogram of a simple reversible electrode reaction $\mathrm{Ox}_{(\text {aq) }}+n \mathrm{e}^{-} \rightleftarrows \operatorname{Red}_{(\mathrm{aq})}$ consists of two apparently symmetric peaks: forward/reduction and reverse/reoxidation. The peaks symmetry disappears as the electrode reaction becomes slower, so that reverse peak completely vanishes for $\kappa<0.05$. The standard rate constant, as well as the electron transfer coefficients ( $\alpha$ and $\beta$ ), can be estimated by the variation of pulse time in ADMPV, i.e. from the slope of linear dependence of the peak currents ratio on the logarithm of pulse time. Therefore, visual inspection of the ADMPV response (i.e. "the voltammogram symmetry") enables us to estimate the electrochemical reversibility of the system, as well as the electron transfer coefficient. The advantage of this technique (using the SMDE) is the fact that the whole experiment (electro- reduction and-oxidation) is taken at the same mercury drop, which is especially useful if the product of electrode reaction is unstable. Also, during a single potential cycle, the electrode reaction is driven in both cathodic and anodic directions, further providing an insight into the electrochemical mechanism.

\section{REFERENCES}

[1] A. Molina, J. Gonzalez, Pulse voltammetry in physical electrochemistry and electroanalysis. Theory and Applications, Springer, Berlin, 2016. https://doi.org/10.1007/978-3-319-21251-7

[2] C. Batchelor-McAuley, E. Kätelhön, E. O. Barnes, R. G. Compton, E. Laborda, A. Molina, ChemistryOpen 2015, 4, 224-260.

https://doi.org/10.1002/open.201500042

[3] V. Mirčeski, R. Gulaboski, M. Lovrić, I. Bogeski, R. Kappl, M. Hoth, Electroanalysis 2013, 25, 2411-2422. https://doi.org/10.1002/elan.201300369

[4] J. Wang, Analytical electrochemistry 2nd ed., Wiley, New York, 2001.

[5] A. J. Bard, L. R. Faulkner, Electrochemical Methods. Fundamental and Applications, Wiley, New York, 2001.

[6] V. Mirčeski, Š. Komorsky-Lovrić, M. Lovrić, Squarewave Voltammetry. Theory and Application, Springer, Berlin, 2007.

https://doi.org/10.1007/978-3-540-73740-7

[7] Z. Stojek and M. Lovrić in Electroanalytical Methods. Guide to Experiments and Applications, (Ed.: F. Scholz), Springer, Berlin, 2010, pp. 117-127.
[8] A. Molina, M. M. Moreno, C. Serna, L. Camacho, Electrochem. Commun. 2001, 3, 324-329. https://doi.org/10.1016/S1388-2481(01)00161-8

[9] A. Molina, M. M. Moreno, M. Lopez-Tenes, C. Serna, Electrochem. Commun. 2002, 4, 457-461. https://doi.org/10.1016/S1388-2481(02)00344-2

[10] C. Serna, A. Molina, M. M. Moreno, M. Lopez-Tenes, J. Electroanal. Chem. 2003, 546, 97-108. https://doi.org/10.1016/S0022-0728(03)00155-4

[11] M. Lopez-Tenes, A. Molina, C. Serna, M. M. Moreno, J. Gonzalez, J. Electroanal. Chem. 2007, 603, 249-259. https://doi.org/10.1016/j.jelechem.2007.02.011

[12] E. Laborda, E. I. Rogers, F. Martinez-Ortiz, A. Molina, R. G. Compton, Electroanalysis 2010, 22, 2784-2793. https://doi.org/10.1002/elan.201000328

[13] E. Laborda, F. Martinez-Ortiz, A. Molina, Electrochim. Acta 2011, 56, 5335-5342. https://doi.org/10.1016/j.electacta.2011.03.132

[14] A. Molina, J. Gonzalez, E. Laborda, R. G. Compton, Int. J. Electrochem. Sci. 2012, 7, 5765-5778.

[15] R. K. Zlatev, M. S. Stoytcheva, B. V. Salas, J.-P. Magnin, P. Ozil, Electrochem. Commun. 2006, 8, 1699-1706.

https://doi.org/10.1016/j.elecom.2006.07.041

[16] R. Zlatev, M. Stoytcheva, B. Valdez, J.-P. Magnin, Z. Velkova, ECS Trans. 2008, 13, 57-63.

http://dx.doi.org/10.1149/1.3002808

[17] J. A. Valera, R. Zlatev, M. Stoytcheva, B. Valdez, M. Carrillo, ECS Trans. 2010, 28, 99-108. https://doi.org/10.1149/1.3491282

[18] R. Zlatev, M. Stoytcheva, B. Valdez, Electroanalysis 2010, 22, 1671-1674. https://doi.org/10.1002/elan.201000090

[19] R. Zlatev, M. Stoytcheva, B. Valdez, Electroanalysis 2018, 30, 1902-1905.

https://doi.org/10.1002/elan.201800291

[20] B. Speiser in Electroanalytical Chemistry, Vol. 19, (Eds.: A. J. Bard, I. Rubinstein.), Dekker, New York, 1996, pp. 2-99.

[21] E. Laborda, E. I. Rogers, F. Martinez-Ortiz, J. G. Limon-Petersen, N. V. Rees, A. Molina, R. G. Compton, J. Electroanal. Chem. 2009, 634, 1-10. https://doi.org/10.1016/j.jelechem.2009.06.022

[22] A. Molina, F. Martinez-Ortiz, E. Laborda, R. G. Compton, J. Electroanal. Chem. 2010, 648, 67-77. https://doi.org/10.1016/j.jelechem.2010.06.019 